\title{
Predictive factors of mortality within 30 days in patients with nonvariceal upper gastrointestinal bleeding
}

Yoo Jin Lee ${ }^{1,}$, Bo Ram Min ${ }^{1,}$, Eun Soo Kim ${ }^{1}$, Kyung Sik Park ${ }^{1}$, Kwang Bum Cho ${ }^{1}$, Byoung Kuk Jang ${ }^{1}$, Woo Jin Chung ${ }^{1}$, Jae Seok Hwang ${ }^{1}$, and Seong Woo Jeon ${ }^{2}$

${ }^{1}$ Division of Gastroenterology and Hepatology, Department of Internal Medicine, Keimyung University School of Medicine, Daegu; ${ }^{2}$ Division of Gastroenterology and Hepatology, Department of Internal Medicine, Kyungpook National University School of Medicine, Daegu, Korea

Received: April 3, 2014

Revised : July 28, 2014

Accepted: November 13, 2014

Correspondence to

Eun Soo Kim, M.D.

Division of Gastroenterology and Hepatology, Department of Internal Medicine, Keimyung University School of Medicine, 56 Dalseong-ro, Jung-gu, Daegu 41931, Korea

Tel: +82-53-250-8096

Fax: $+82-53-250-7442$

E-mail: dandy813@hanmail.net

*These authors contributed equally to this work.
Background/Aims: Nonvariceal upper gastrointestinal bleeding (NVUGIB) is a common medical emergency that can be life threatening. This study evaluated predictive factors of 30 -day mortality in patients with this condition.

Methods: A prospective observational study was conducted at a single hospital between April 2010 and November 2012, and 336 patients with symptoms and signs of gastrointestinal bleeding were consecutively enrolled. Clinical characteristics and endoscopic findings were reviewed to identify potential factors associated with 30 -day mortality.

Results: Overall, 184 patients were included in the study (men, 79.3\%; mean age, 59.81 years), and 16 patients died within 30 days (8.7\%). Multivariate analyses revealed that comorbidity of diabetes mellitus (DM) or metastatic malignancy, age $\geq 65$ years, and hypotension (systolic pressure $<90 \mathrm{mmHg}$ ) during hospitalization were significant predictive factors of 30 -day mortality.

Conclusions: Comorbidity of DM or metastatic malignancy, age $\geq 65$ years, and hemodynamic instability during hospitalization were predictors of 30 -day mortality in patients with NVUGIB. These results will help guide the management of patients with this condition.

Keywords: Gastrointestinal hemorrhage; Mortality; Prognosis; Comorbidity

\section{INTRODUCTION}

Nonvariceal upper gastrointestinal bleeding (NVUGIB) is the most common medical emergency, and is a considerable clinical and economic burden [1]. Although the mortality rate in patients with NVUGIB has declined dramatically in recent years due to the development of proton pump inhibitors and endoscopic therapy, it remains high at $5 \%$ to $10 \%[2,3]$. A recent international consensus on NVUGIB emphasized the importance of early risk stratification for rebleeding and mortality [4]. Several risk factors for this condition have been proposed, and of these, rebleeding was a significant predictor of mortality [5-7]. Other predictive factors include advanced age, liver cirrhosis, chronic kidney disease, advanced neoplasia, low hemoglobin level, cardiac failure, and hemodynamic instability $[1,8]$. Early recognition of the risk of death would facilitate differentiation between high- and low-risk patients. Close monitoring and careful management of high-risk patients could improve 
their prognosis. Therefore, predictors of mortality in patients with NVUGIB are clinically valuable. The aim of this prospective study was to investigate potential predictors associated with 30-day mortality in patients admitted to the emergency unit for this condition.

\section{METHODS}

\section{Patients}

This prospective, single-center, observational study was conducted at a tertiary hospital in Daegu, Korea(Keimyung University Dongsan Hospital), and was approved by the institutional review board of the university (No. 11-294). Written informed consent was obtained from all of the subjects enrolled in the study. This study is registered in the World Health Organization International Clinical Trials Registry Platform (KCToooo514).

Adult patients ( $>18$ years), clinically diagnosed with gastrointestinal bleeding between April 2010 and November 2012, were enrolled. Patients were excluded if they did not complete at least 30 days of follow-up, if the bleeding source was varices or gastric cancer, if bleeding was associated with endoscopic procedures such as endoscopic mucosal resection, or if bleeding occurred in the lower gastrointestinal tract. The following information was documented prospectively: patient data (age, sex, date of admission, and endoscopy results); historical data (presenting symptoms, previous history of gastrointestinal bleeding, or peptic ulcer disease); social history such as current smoking status, alcohol consumption (heavy alcoholics were defined as women and men who consumed more than 40 and $60 \mathrm{~g}$ alcohol per day, respectively, for more than 5 years) [9]; physical findings (results of nasogastric tube aspiration, results of rectal examination, and initial hemodynamic status); initial laboratory data (hemoglobin and blood urea levels, platelet count, and nitrogen prothrombin time); and comorbidities including hypertension, diabetes mellitus (DM), ischemic heart disease, cerebrovascular disease, heart failure, liver cirrhosis (liver cirrhosis was defined according to clinical, laboratory, and radiologic data but not liver biopsy results because biopsy was not performed) [10], chronic kidney disease (defined as patients with an estimated glomerular filtration rate $<60 \mathrm{~mL} /$ min for at least 3 months calculated using the four-vari- able Modification of Diet in Renal Disease Study equation) [11], metastatic malignancy, and peripheral vascular disease. Hemodynamic instability was defined as tachycardia with a heart rate $>100$ beats per minute and hypotension with a systolic pressure $<90 \mathrm{mmHg}$ upon admission and during hospitalization. Medication history (antiplatelet agents, vitamin $\mathrm{K}$ antagonists, nonsteroidal anti-inflammatory drugs [NSAIDs], steroids, and proton pump inhibitors) was also recorded if patients had taken the aforementioned drugs 1 week before the first bleeding event. Patients were managed according to recent consensus recommendations, including endoscopic and pharmacologic management as well as transfusion [4]. All of the patients were intravenously administered proton pump inhibitors. In addition, each patient's risk of bleeding was assessed by a Rockall score and a Blatchford score. The total Rockall score includes both endoscopic and nonendoscopic variables, whereas the Blatchford score is only based on nonendoscopic variables $[6,7]$. High-risk patients were defined as those with a total Rockall score $\geq 5$, indicating an estimated mortality rate of $40 \%$ [6].

\section{Endoscopic procedures}

All of the endoscopic procedures for acute upper gastrointestinal bleeding (UGIB) were performed within 24 hours after arrival at the hospital. Procedures were performed by experienced endoscopists who had previously conducted > 1,000 endoscopies. Urgent endoscopy was defined as an endoscopy procedure conducted within 12 hours of admission [4]. High-risk patients underwent urgent endoscopy according to a strict hospital protocol. However, in patients who showed unfavorable clinical conditions for endoscopy or in those who arrived at night or during the weekend, the decision to perform this procedure was made by the individual endoscopist. Endoscopic variables included the cause of UGIB and location of the lesion. Lesions are either located in the body of the stomach, where the risk of bleeding is high due to numerous supplying vessels, or in other parts of the stomach [12]. Unidentified causes of bleeding were defined as the presence of blood in the stomach without any identifiable source of bleeding [3]. In patients with ulcers, bleeding activity at the ulcer base was classified according to the Forrest classification [13]. Forrest I, IIa, and IIb represent high-risk bleeding stigmata (HRBS) 
$[3,6]$. Endoscopic findings were reviewed and adjusted by two expert endoscopists (YJL and ESK) to improve interobserver agreement.

\section{Outcomes}

The primary outcome was mortality within 30 days of admission. Patients were advised to return for an examination at 4 and 12 weeks after discharge. For discharged or deceased patients, the relevant data from hospital records were extracted by investigators. Patients with hospital record data that did not satisfy the inclusion criteria were excluded. A 30-day rebleeding episode was defined as the onset of new hematemesis or hematochezia with hypovolemic shock, or a greater than $2 \mathrm{~g} / \mathrm{dL}$ decrease in blood hemoglobin levels after a 24-hour period of stable vital signs within 30 days of admission $[3,14]$. All of the rebleeding episodes were confirmed with endoscopy. Bleeding-related 30-day mortality included death from irreversible hypovolemic shock during surgery for uncontrolled bleeding or endoscopy-related mortality occurring within 30 days of the index-bleeding episode. Nonbleeding-related 30-day mortality was defined as death from cardiac, pulmonary or cerebrovascular disease, liver or kidney failure, and malignant disease [15].

\section{Statistical analysis}

Statistical analyses were performed using SPSS version 18.0 (SPSS Inc., Chicago, IL, USA). The Student $t$ test was used for comparison of continuous variables. Categorical variables were compared using Fisher exact test or a chi-square test. Independent risk factors for 30-day mortality were assessed by multivariate logistic regression analysis. An odds ratio (OR) and 95\% confidence interval (CI) was calculated for each independent factor. A two-tailed $p<0.05$ was considered statistically significant.

\section{RESULTS}

\section{Patient characteristics}

During the study period, 336 patients with gastrointestinal bleeding were admitted, and 184 (54.8\%) met the inclusion criteria (Fig. 1). A total of 144 patients were excluded from the study because the source of bleeding was varices ( 82 patients), the lower gastrointestinal tract

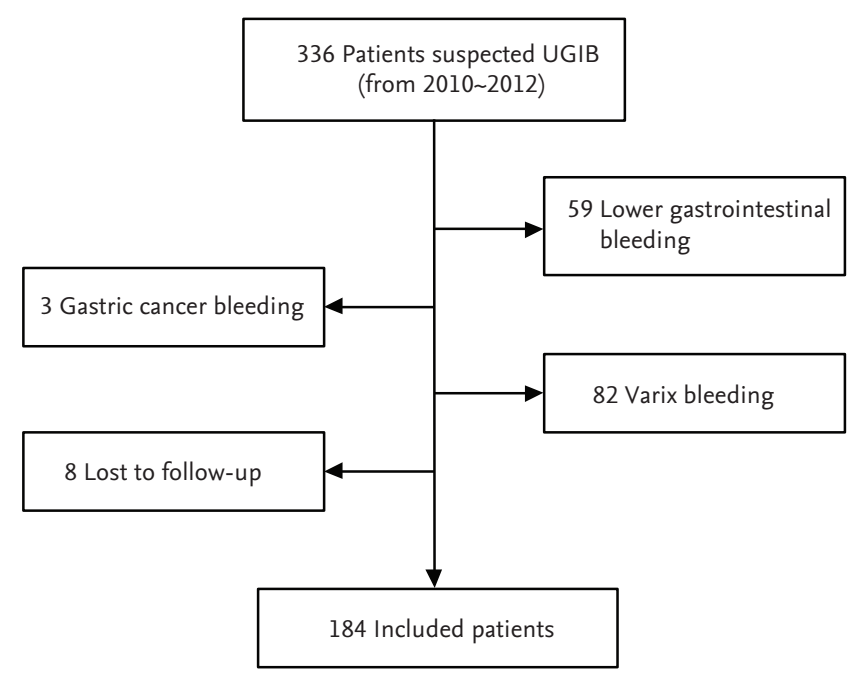

Figure 1. Flow chart of patient selection in the study. UGIB, upper gastrointestinal bleeding.

(59 patients), or gastric cancer (3 patients). Eight patients were lost to follow-up. Patient characteristics are shown in Table 1. The mean age was 59.81 years, and $79.3 \%$ of patients were male. The most frequently presenting symptom was hematemesis (50.0\%). A total of 48 patients (26.1\%) had a previous history of gastrointestinal bleeding, and 60 (32.6\%) had a previous history of peptic ulcer disease. The most common comorbidity was hypertension (82 patients, $44.6 \%$ ), followed by DM (44 patients, $23.9 \%$ ). With regard to concomitant use of drugs that could have been related to bleeding, 53 patients (28.8\%) used antiplatelet agents including aspirin, clopidogrel, or cilostazol, and 37 patients (20.1\%) used NSAIDs. As outlined in Table 2, the mean serum level of hemoglobin upon admission was $8.97 \mathrm{~g} / \mathrm{dL}$. The percentage of patients with tachycardia (heart rate $>100$ beats per minute) and hypotension (systolic pressure < $90 \mathrm{mmHg}$ ) during hospitalization was $33.7 \%$ and $22.8 \%$, respectively. The percentage of patients with positive nasogastric tube aspiration and digital rectal examination was 68.9\% (122/177) and 68.7\% (123/179), respectively. Packed red blood cells were transfused in 111 patients (60.3\%), and the mean number of units transfused was $2.36 \pm$ 3.69. The mean total Rockall score was $4.62 \pm 2.00$, and 96 patients $(52.2 \%)$ had a score $\geq 5$, indicating a high risk of mortality. The mean Blatchford score was 11.49 \pm 3.38 .

An urgent endoscopy ( $<12$ hours after admission) was conducted in 121 patients $(65.8 \%)$, whereas the remaining patients $(63,34.2 \%)$ underwent endoscopy 12 to 24 
Table 1. General characteristics of patients with nonvariceal upper gastrointestinal bleeding $(n=184)$

\begin{tabular}{|c|c|}
\hline Factor & Value \\
\hline Male sex & $146(79 \cdot 3)$ \\
\hline Age, yr & $59.81 \pm 15.80$ \\
\hline \multicolumn{2}{|l|}{ Bleeding related symptoms } \\
\hline Hematemesis & $92(50.0)$ \\
\hline Tarry stool & $77(41.8)$ \\
\hline Hematochezia & $12(6.5)$ \\
\hline Acute onset anemia & $3(1.6)$ \\
\hline Heavy alcoholics & $72(39.1)$ \\
\hline Current smoker & $68(37.0)$ \\
\hline Past history of gastrointestinal bleeding & $48(26.1)$ \\
\hline Past history of peptic ulcer disease & $60(32.6)$ \\
\hline \multicolumn{2}{|l|}{ Comorbidities } \\
\hline Hypertension & $82(44 \cdot 6)$ \\
\hline Diabetes mellitus & $44(23.9)$ \\
\hline Liver cirrhosis & $30(16.3)$ \\
\hline Chronic kidney disease & $28(15.2)$ \\
\hline Cerebrovascular disease & $26(14.1)$ \\
\hline Heart failure & $27(14.7)$ \\
\hline Cardiovascular disease & $22(12.0)$ \\
\hline Metastatic malignancy & $8(4 \cdot 3)$ \\
\hline Peripheral vascular disease & $4(2.2)$ \\
\hline \multicolumn{2}{|l|}{ Use of medication } \\
\hline Antiplatelet agents & $53(28.8)$ \\
\hline NSAIDs & $37(20.1)$ \\
\hline Vitamin $\mathrm{K}$ antagonist & $8(4 \cdot 3)$ \\
\hline PPI co-medication & $7(3.8)$ \\
\hline
\end{tabular}

Values are presented as number (\%) or mean \pm SD.

NSAID, nonsteroidal anti-inflammatory drug; PPI, proton pump inhibitor.

hours after admission. Peptic ulcer was the main cause of bleeding (78.3\%); other causes of bleeding were Mallory-Weiss syndrome (12.5\%), angiodysplasia (1.6\%), and hemorrhagic gastritis (0.5\%). The cause of bleeding in 13 patients $(7.1 \%)$ could not be identified despite the presence of blood in the stomach. Of the 144 patients with peptic ulcer, 78 (54.2\%) showed HRBS (Forrest I, 22.2\%; IIa, 20.8\%; and IIb, 11.1\%) (Table 3).

\section{Clinical results}

The median follow-up period was 196 days (interquar-
Table 2. Clinical data at the time of admission to the hospital for nonvariceal upper gastrointestinal bleeding $(n=184)$

\begin{tabular}{|c|c|}
\hline Factor & Value \\
\hline \multicolumn{2}{|l|}{ Initial vital sign } \\
\hline $\mathrm{SBP}, \mathrm{mmHg}$ & $117.58 \pm 22.79$ \\
\hline Heart rate, beat/min & $88.75 \pm 17.97$ \\
\hline \multicolumn{2}{|l|}{ Initial laboratory data } \\
\hline Hemoglobin, g/dL & $8.97 \pm 2.95$ \\
\hline Platelets, $\times 10^{9} / \mathrm{L}$ & $253 \pm 117$ \\
\hline Blood nitrogen urea, mg/dL & $42.54 \pm 28.53$ \\
\hline Prothrombin time, sec & $13.29 \pm 8.60$ \\
\hline Positive nasogastric tube aspiration ${ }^{\mathrm{a}}$ & $122 / 177(68.9)$ \\
\hline Positive rectal examination ${ }^{b}$ & $123 / 179(68.7)$ \\
\hline Transfusion requirement & $111(60.3)$ \\
\hline Transfusion requirement, no. of unit & $2.36 \pm 3.69$ \\
\hline $\begin{array}{l}\text { Tachycardia (heart rate > } 100 \text { beat/min) } \\
\text { during the hospital stay }\end{array}$ & $62(33.7)$ \\
\hline $\begin{array}{l}\text { Hypotension }(\mathrm{SBP}<90 \mathrm{mmHg}) \\
\text { during the hospital stay }\end{array}$ & $42(22.8)$ \\
\hline Blatchford score & $11.49 \pm 3.38$ \\
\hline Rockall score & $4.62 \pm 2.00$ \\
\hline
\end{tabular}

Values are presented as mean $\pm \mathrm{SD}$ or number (\%).

SBP, systolic blood pressure.

${ }^{a}$ Nasogastric tube was performed in 177 patients.

${ }^{\mathrm{b}}$ Rectal examination was performed in 179 patients.

tile range, 77 to 404), and the overall number of deaths during this period was 38 (20.7\%). The number of deaths within 30 days was 16 (8.7\%) (Table 4). Fig. 2 shows the survival curve of patients with NVUGIB over a follow-up period of 30 days. Among patients who died within 30 days, half $(8 / 16,50.0 \%)$ died during the first 7 days; the 7-day survival probability was estimated to be $96.6 \%$. The causes of 30-day mortality are presented in Table 5. Bleeding-related death within 30 days occurred in five patients (31.2\%), and all of them died within the first 7 days. The deaths of the remaining 11 patients (68.8\%) were associated with their comorbidities. Cardiovascular events including heart failure or acute myocardial infarction were the most frequent nonbleeding-related causes of 30-day mortality (seven patients, $43.8 \%$ ), followed by liver failure (two patients, $12.5 \%$ ), brain hemorrhage (one patient, 6.3\%), and metastatic cancer progression (one patient, 6.3\%). Overall, rebleeding occurred in 38 patients $(20.7 \%)$ during the follow-up period, and rebleeding within 30 days of admission occurred in 27 
Table 3. Endoscopic features of patients with nonvariceal upper gastrointestinal bleeding $(n=184)$

\begin{tabular}{lc}
\hline Factor & No. $(\%)$ \\
\hline Urgent endoscopy $^{\mathrm{a}}$ & $121(65.8)$ \\
\hline Endoscopy finding & $144(78.3)$ \\
\hline Peptic ulcer disease & $23(12.5)$ \\
\hline Mallory-Weiss syndrome & $3(1.6)$ \\
\hline Angiodysplasia & $1(0.5)$ \\
\hline Hemorrhagic gastritis & $13(7.1)$ \\
\hline No evidence of upper gastrointestinal & \\
\hline bleeding & $89(48.4)$ \\
\hline Endoscopy lesion location & $46(25.0)$ \\
\hline Cardia, angle, antrum & $35(19.0)$ \\
\hline Body & $14(7.6)$ \\
\hline Duodenum & \\
\hline No specific lesion & $32 / 144(22.2)$ \\
\hline Forrest classification ${ }^{b}$ & $30 / 144(20.8)$ \\
\hline I & $16 / 144(11.1)$ \\
\hline IIa & $40 / 144(27.8)$ \\
\hline IIb & $26 / 144(18.1)$ \\
\hline IIc & \\
\hline III & \\
\hline
\end{tabular}

${ }^{\mathrm{a}}$ Endoscopy which was performed within 12 hours of admission.

${ }^{\mathrm{b}}$ Classified in 144 patients who had ulcers.

Table 4. Clinical outcomes of patients with nonvariceal upper gastrointestinal bleeding $(n=184)$

\begin{tabular}{ll}
\hline Factor & Value \\
\hline Rebleeding & \\
During follow-up period $^{\mathrm{a}}$ & $38(20.7)$ \\
Within 30 days $^{\text {Death }}$ & $27(14.7)$ \\
\hline During follow-up period & \\
Within 30 days & $38(20.7)$ \\
Hospital stay, day & $16(8.7)$ \\
\hline
\end{tabular}

Values are presented as number (\%) or mean \pm SD.

${ }^{a}$ Median follow-up period of 196 days (interquartile range, 77-404 days).

patients (14.7\%). The median length of hospitalization was 6 days.

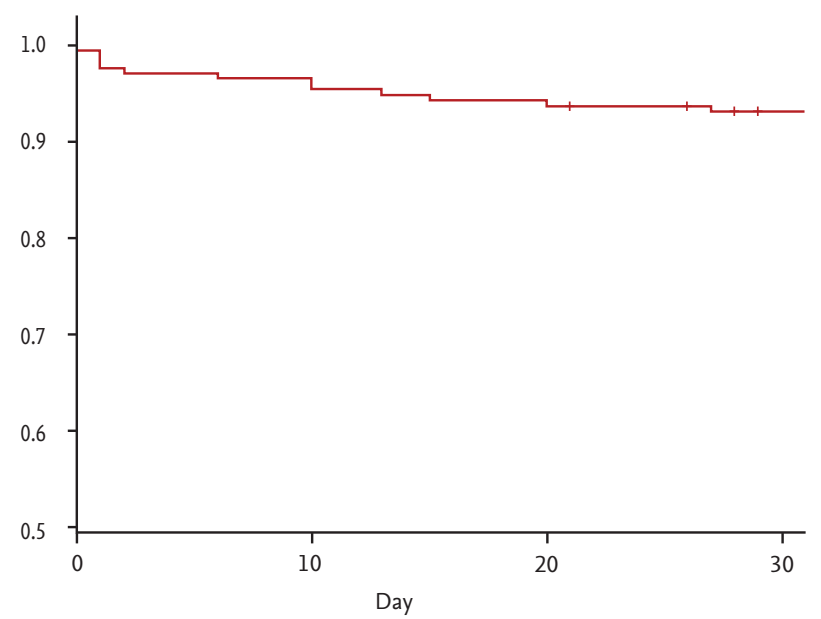

Figure 2. Kaplan-Meier survival curve of 30-day mortality in patients with nonvariceal upper gastrointestinal bleeding. Eight patients died during the first 7 days (cumulative survival, 96.6\%).

\section{Predictive factors for 30-day mortality in patients with NVUGIB}

According to univariate analysis, age $\geq 65$ years $(p=$ $0.009), \mathrm{DM}(p=0.004)$, chronic kidney disease $(p=0.004)$, metastatic malignancy $(p=0.023)$, heart failure $(p=0.016)$, Rockall score $\geq 5(p=0.003)$, tachycardia (heart rate $>100$ beats per minute) during hospitalization $(p<0.001)$, hypotension (systolic pressure $<90 \mathrm{mmHg}$ ) during hospitalization $(p<0.001)$, and rebleeding within 30 days $(p$ $<0.001$ ) were significant risk factors for 30-day mortality. Multivariate logistic regression analysis identified the following variables as independent predictors of increased 30-day mortality in patients with NVUGIB: comorbidity of DM (OR, 12.67; 95\% CI, 1.92 to 83.45; $p=0.008)$ or metastatic malignancy (OR, 29.24; 95\% CI, 2.08 to $411.67 ; p=0.012)$, age $\geq 65$ years $(\mathrm{OR}, 5.06 ; 95 \% \mathrm{CI}$, 1.79 to $32.60 ; p=0.048$ ), and hypotension (systolic pressure < $90 \mathrm{mmHg}$ ) during hospitalization (OR, 16.63; 95\% CI, 2.56 to 107.90; $p=0.003$ ) (Table 6). Predictors for 30day mortality were also analyzed after dividing patients into high- and low-risk bleeding stigmata groups. However, there were no independent risk factors for 30-day mortality according to risk stratification by Forrest classification (Supplementary Tables 1 and 2). 
Table 5. Characteristics of the 16 patients who died within 30 days

\begin{tabular}{|c|c|c|c|c|c|c|c|c|c|}
\hline Patient & Age/Sex & $\begin{array}{l}\text { Rockall } \\
\text { score }\end{array}$ & $\begin{array}{l}\text { Blatchford } \\
\text { score }\end{array}$ & $\begin{array}{l}\text { Forrest } \\
\text { class }\end{array}$ & $\begin{array}{l}\text { Endoscopy } \\
\text { finding }\end{array}$ & $\begin{array}{l}\text { Bleeding } \\
\text { lesion }\end{array}$ & Comorbidities & $\begin{array}{l}\text { Days from } \\
\text { admission } \\
\text { to death }\end{array}$ & $\begin{array}{c}\text { Cause of } \\
\text { death }\end{array}$ \\
\hline 1 & $63 / \mathrm{M}$ & 6 & 13 & $2 \mathrm{~A}$ & GU & Antrum & HTN, CKD & 1 & Rebleeding \\
\hline 2 & $86 / \mathrm{M}$ & 6 & 14 & $1 B$ & GU & UB & CKD, HF & 1 & Rebleeding \\
\hline 3 & $87 / \mathrm{M}$ & 9 & 12 & ${ }_{1 B} B$ & GU \& DU & 2nd & $\begin{array}{l}\text { HTN, DM, CKD, } \\
\text { CVA }\end{array}$ & 1 & Rebleeding \\
\hline 4 & $79 / \mathrm{F}$ & 7 & 13 & $1 \mathrm{~B}$ & $\mathrm{DU}$ & Bulb & HTN, HF & 1 & Rebleeding \\
\hline 5 & $49 / \mathrm{M}$ & 4 & 10 & - & No lesion & - & $\begin{array}{l}\mathrm{HTN}, \mathrm{DM}, \mathrm{HF}, \\
\mathrm{CHB}\end{array}$ & 1 & Liver failure \\
\hline 6 & $86 / \mathrm{M}$ & 7 & 13 & $2 \mathrm{~B}$ & $\mathrm{GU}$ & Angle & $\mathrm{DM}$ & 2 & AMI \\
\hline 7 & $72 / \mathrm{F}$ & 5 & 13 & 3 & $\mathrm{GU}$ & Antrum & HTN, DM & 2 & $\begin{array}{l}\text { Brain } \\
\text { hemorrhage }\end{array}$ \\
\hline 8 & $69 / M$ & 11 & 8 & $2 \mathrm{~A}$ & DU & Bulb & $\begin{array}{l}\text { HTN, CVA, } \\
\text { metastatic } \\
\text { malignancy }\end{array}$ & 6 & Rebleeding \\
\hline 9 & $76 / \mathrm{F}$ & 4 & 14 & $2 \mathrm{C}$ & GU & Antrum & $\begin{array}{l}\text { HTN, DM, CVA, } \\
\text { HF }\end{array}$ & 10 & $\mathrm{HF}$ \\
\hline 10 & $80 / F$ & 5 & 12 & $2 B$ & $\mathrm{DU}$ & 2nd & HTN & 10 & $\mathrm{HF}$ \\
\hline 11 & $63 / \mathrm{M}$ & 5 & 7 & - & No lesion & - & $\begin{array}{l}\text { Metastatic } \\
\text { malignancy }\end{array}$ & 13 & $\begin{array}{l}\text { Cancer } \\
\text { progression }\end{array}$ \\
\hline 12 & $71 / \mathrm{M}$ & 7 & 12 & $2 \mathrm{~A}$ & GU & MB & $\begin{array}{l}\text { HTN, DM, CKD, } \\
\text { CVA }\end{array}$ & 15 & AMI \\
\hline 13 & $42 / \mathrm{M}$ & 6 & 10 & $1 \mathrm{~B}$ & MWS & Cardia & $\begin{array}{l}\text { LC, CKD, } \\
\text { metastatic } \\
\text { malignancy }\end{array}$ & 20 & Liver failure \\
\hline 14 & $72 / \mathrm{M}$ & 7 & 12 & 3 & GU & Antrum & $\begin{array}{l}\text { HTN, DM, CKD } \\
\text { CVD, HF }\end{array}$ & 24 & AMI \\
\hline 15 & $69 / \mathrm{M}$ & 5 & 13 & $2 \mathrm{C}$ & GU & $\mathrm{LB}$ & DM, CVA & 27 & $\mathrm{HF}$ \\
\hline 16 & $69 / \mathrm{M}$ & 6 & 15 & $2 \mathrm{C}$ & GU & Cardia & $\begin{array}{l}\mathrm{HTN}, \mathrm{DM}, \mathrm{HF}, \\
\text { CKD }\end{array}$ & 30 & $\mathrm{HF}$ \\
\hline
\end{tabular}

GU, gastric ulcer; HTN, hypertension; CKD, chronic kidney disease; UB, upper body; HF, heart failure; DU, duodenal ulcer; 2nd, duodenum 2nd portion; DM, diabetes mellitus; CVA, cerebrovascular attack; CHB, chronic hepatitis B; AMI, acute myocardial infarction; MB, mid body; MWS, Mallory-Weiss syndrome; LC, liver cirrhosis; LB, lower body.

\section{DISCUSSION}

In our study, we found that age ( $\geq 65$ years), comorbidity of DM or metastatic malignancy, and hypotension (systolic pressure $<90 \mathrm{mmHg}$ ) during hospitalization were independently associated with mortality within 30 days. The 30-day mortality rate was $8.7 \%$, which was slightly higher than the rates of $5.4 \%$ and $4.5 \%$ reported by the Canadian registry [16] and Italian database study [1], respectively. Although the reason for the higher mor- tality rate in our study was not clear, it may have been due to differences in the study populations. This study was conducted at a tertiary hospital; therefore, patients' conditions may have been more severe, which may have contributed to poor patient outcomes and a higher mortality rate. On the other hand, the mortality rate was lower than that in a recent study from England that reported a 28 -day mortality rate of $13.1 \%$ [17].

It is notable that half of all 30-day deaths occurred within the first 7 days, and all rebleeding-related 30-day 
Table 6. Predictive factors for 30-day mortality $(n=184)$

\begin{tabular}{|c|c|c|c|c|c|}
\hline \multirow[b]{2}{*}{ Factor } & \multicolumn{3}{|c|}{ Univariate analysis } & \multicolumn{2}{|c|}{ Multivariate analysis } \\
\hline & $\begin{array}{l}\text { 30-Day death }(+) \\
(n=16)\end{array}$ & $\begin{array}{l}\text { 30-Day death }(-) \\
\quad(n=168)\end{array}$ & $p$ value & Odds ratio (95\% CI) & $p$ value \\
\hline Male sex & $14(87.5)$ & $133(79.2)$ & 1.000 & - & - \\
\hline Age $\geq 65$ yr & $11(68.7)$ & $65(38.7)$ & $0.009^{\mathrm{a}}$ & $5.06(1.79-32.60)$ & $0.048^{a}$ \\
\hline \multicolumn{6}{|l|}{ Initial vital signs } \\
\hline $\mathrm{SBP}<90 \mathrm{mmHg}$ & 0 & $13(7.7)$ & 0.608 & - & - \\
\hline Heart rate $>100$ beat $/ \mathrm{min}$ & $5(31.2)$ & $43(25 \cdot 6)$ & 0.567 & - & - \\
\hline \multicolumn{6}{|l|}{ Initial laboratory data } \\
\hline Hemoglobin, g/dL & $8.60 \pm 3.29$ & $9.0 \pm 2.93$ & 0.581 & - & - \\
\hline Platelets, $\times 10^{9} / \mathrm{L}$ & $287 \pm 187$ & $250 \pm 109$ & 0.445 & - & - \\
\hline Blood nitrogen urea, mg/dL & $58.81 \pm 32.30$ & $40.99 \pm 27.76$ & 0.071 & - & - \\
\hline Prothrombin time, sec & $16.47 \pm 10.75$ & $13.01 \pm 8.36$ & 0.242 & - & - \\
\hline $\begin{array}{l}\text { Severe bleeding related symptoms } \\
\text { (hematemesis, hematochezia) }\end{array}$ & $9(56.2)$ & $93(55 \cdot 4)$ & 0.945 & - & - \\
\hline Positive nasogastric tube aspiration ${ }^{\mathrm{b}}$ & $12(75.0)$ & $110(65 \cdot 5)$ & 0.779 & - & - \\
\hline Positive rectal examination ${ }^{c}$ & $14(87.5)$ & $109(64.9)$ & 0.089 & - & - \\
\hline Heavy alcoholics & $6(37 \cdot 5)$ & $66(39 \cdot 3)$ & 0.889 & - & - \\
\hline Current smoker & $5(31.2)$ & $63(37 \cdot 5)$ & 0.621 & - & - \\
\hline Past history of gastrointestinal bleeding & $1(6.2)$ & $47(28.0)$ & 0.074 & - & - \\
\hline Past history of peptic ulcer disease & $5(31.2)$ & $55(32.7)$ & 0.903 & - & - \\
\hline Transfusion requirement & $13(81.2)$ & $98(58.3)$ & 0.073 & - & - \\
\hline Red cell transfusion unit & $4.56 \pm 5.62$ & $2.14 \pm 3.39$ & 0.110 & - & - \\
\hline \multicolumn{6}{|l|}{ Comorbidities } \\
\hline Hypertension & $11(68.8)$ & $71(42 \cdot 3)$ & 0.052 & - & - \\
\hline Diabetes mellitus & $9(56.2)$ & $35(20.8)$ & $0.004^{\mathrm{a}}$ & $12.67(1.92-83.45)$ & $0.008^{a}$ \\
\hline Cardiovascular disease & $1(6.2)$ & $21(12.5)$ & 0.698 & - & - \\
\hline Liver cirrhosis & $1(6.2)$ & $29(17 \cdot 3)$ & 0.477 & - & - \\
\hline Chronic kidney disease & $7(43.8)$ & $21(12.5)$ & $0.004^{\mathrm{a}}$ & $1.18(0.15-9.59)$ & 0.876 \\
\hline Cerebrovascular disease & $5(31.2)$ & $21(12.5)$ & 0.055 & - & - \\
\hline Heart failure & $6(37.5)$ & $21(12.5)$ & $0.016^{\mathrm{a}}$ & $1.96(0.33-11.76)$ & 0.461 \\
\hline Metastatic malignancy & $3(18.8)$ & $5(3.0)$ & $0.023^{\mathrm{a}}$ & $29.24(2.08-411.67)$ & $0.012^{\mathrm{a}}$ \\
\hline Peripheral vascular disease & $1(6.2)$ & $3(1.8)$ & 0.307 & - & - \\
\hline \multicolumn{6}{|l|}{ Medication } \\
\hline Antiplatelet agents & $7(43.8)$ & $46(27 \cdot 4)$ & 0.245 & - & - \\
\hline Vitamin K antagonist & $2(12.5)$ & $6(3.6)$ & 0.146 & - & - \\
\hline Nonsteroidal anti-inflammatory drug & $4(25 \cdot 0)$ & $33(19.6)$ & 0.533 & - & - \\
\hline PPI comedication & $1(6.2)$ & $6(3.6)$ & 1.000 & - & - \\
\hline Blatchford score $\geq 12$ & $12(75 \cdot 0)$ & $99(58.9)$ & 0.209 & - & - \\
\hline Rockall score $\geq 5$ & $14(87.5)$ & $82(48.8)$ & $0.003^{\mathrm{a}}$ & $1.11(0.15-8.49)$ & 0.920 \\
\hline Endoscopy finding & - & - & 1.000 & - & - \\
\hline Peptic ulcer disease & $13(81.2)$ & $131(78.0)$ & - & & - \\
\hline Nonpeptic ulcer disease & $3(18.8)$ & $37(22.0)$ & - & - & - \\
\hline
\end{tabular}


Table 6. Continued

\begin{tabular}{|c|c|c|c|c|c|}
\hline \multirow[b]{2}{*}{ Factor } & \multicolumn{3}{|c|}{ Univariate analysis } & \multicolumn{2}{|c|}{ Multivariate analysis } \\
\hline & $\begin{array}{l}\text { 30-Day death }(+) \\
\qquad(\mathrm{n}=16)\end{array}$ & $\begin{array}{l}\text { 30-Day death }(-) \\
\quad(n=168)\end{array}$ & $p$ value & Odds ratio (95\% CI) & $p$ value \\
\hline Endoscopy lesion location & - & - & 0.765 & - & - \\
\hline Body & $3(18.8)$ & $44(26.2)$ & - & - & - \\
\hline Other than body & $13(81.2)$ & $124(73.8)$ & - & - & - \\
\hline $\begin{array}{l}\text { High risk endoscopic stigmata } \\
\text { (Forrest I, IIa, and IIb) }^{\mathrm{d}}\end{array}$ & $8(61.5)$ & $70(53.4)$ & 0.576 & - & - \\
\hline $\begin{array}{l}\text { Tachycardia (heart rate > 100 beat/min) } \\
\text { during the admission }\end{array}$ & $12(75.0)$ & $50(29.8)$ & $<0.001^{\mathrm{a}}$ & $3.59(0.60-21.40)$ & 0.161 \\
\hline $\begin{array}{l}\text { Hypotension }(\mathrm{SBP}<90 \mathrm{mmHg}) \text { during the } \\
\text { admission }\end{array}$ & $11(68.8)$ & $31(18.5)$ & $<0.001^{\mathrm{a}}$ & $16.63(2.56-107.90)$ & $0.003^{\mathrm{a}}$ \\
\hline Rebleeding within 30 days & $8(50.0)$ & $19(11.3)$ & $<0.001^{\mathrm{a}}$ & $6.40(0.80-51.07)$ & 0.080 \\
\hline Hospital stay, day & $6.75 \pm 6.66$ & $5.88 \pm 5.74$ & 0.566 & - & - \\
\hline
\end{tabular}

Values are presented as number (\%) or mean $\pm \mathrm{SD}$.

CI, confidence interval; SBP, systolic blood pressure; PPI, proton pump inhibitor.

${ }^{\text {a }}$ Significant values with $p<0.05$.

${ }^{\mathrm{b}}$ Nasogastric tube was performed in 177 patients.

${ }^{\mathrm{c}}$ Rectal examination was performed in 179 patients.

${ }^{\mathrm{d} C l a s s i f i e d ~ i n ~} 144$ patients who had ulcers.

deaths also occurred during this period, suggesting that rebleeding was the cause of early death (within 7 days), whereas comorbidities were responsible for later deaths. These findings are consistent with those from the Italian database study in which early death was mainly related to hemorrhage and late death was associated with patients' comorbidities [18].

In our study, underlying comorbidities were the cause of death in more than half of patients $(11 / 16,68.8 \%)$. Interestingly, 30-day rebleeding was not an independent risk factor of 30-day mortality on multivariate analysis after adjusting for confounding factors, which is in accordance with previous findings [19-21]. Sung et al. [21] reported that 10,429 patients with NVUGIB had $6.2 \%$ mortality, and bleeding-related death was observed in only $29.2 \%$; in the remaining patients (70.8\%), comorbidities were the cause of death. Therefore, early monitoring of rebleeding in patients with NVUGIB is essential for reducing early death. In patients with comorbidities, long-term monitoring of rebleeding after discharge is an appropriate strategy to reduce mortality.

Advanced age is associated with adverse outcomes such as rebleeding or mortality in UGIB $[2,22]$. Consistent with previous studies, we found that age ( $\geq 65$ years) was an important predictor of 30-day mortality regardless of severity and source of bleeding. It is unclear why the mortality rate in elderly patients with UGIB is so high, but it is likely due to multiple complex factors. For example, elderly patients are more likely to have diverse and complex comorbidities and be more susceptible to physiological changes associated with acute bleeding events than younger patients. Therefore, careful follow-up with intensive monitoring is needed in this population following a bleeding episode.

In addition to older age, certain comorbidities such as metastatic malignancies, renal failure, hepatic disease, and heart failure are associated with a greater risk of rebleeding and mortality in patients with UGIB $[6,7]$. In our study, patients with DM (OR, 12.67; 95\% CI, 1.92 to $83.45 ; p=0.008$ ) had a high risk of 30 -day mortality. There is limited information on how DM contributes to a higher mortality rate in patients with NVUGIB. However, there are several possible explanations. First, although cardiovascular disease was not a predictive factor of 30-day mortality, it accounted for a large proportion of the leading cause of death (7/16 patients) in this study. In general, patients with DM have elevated rates of cardiovascular complications and mortality due 
to impaired coronary microvascular function [23,24]. Second, diabetic angiopathy impairs mucosal integrity, leading to more severe ulcers [25]. Lastly, DM increases susceptibility to acute gastrointestinal injury and affects mucosal healing [26]. In addition to DM, metastatic malignancy was associated with a higher 30-day mortality rate (OR, 29.24; 95\% CI, 2.08 to 411.67; $p=0.012$ ). Critically ill patients with cancer have an overall 30 -day mortality rate of up to $50 \%$ and a high risk of severe sepsis related to immunosuppression caused by the malignancy and its treatment [27]. We hypothesized that patients with metastatic malignancy may have been in poor physiological condition and more vulnerable to acute illness.

In our study, hypotension (systolic pressure $<90 \mathrm{mmHg}$ ) during hospitalization was associated with a high risk of 30-day mortality after adjustment for confounding factors (OR, 16.63; 95\% CI, 2.56 to 107.90; $p=0.003$ ). These findings are in agreement with those from prior reports showing an association between hemodynamic instability from UGIB and mortality [5-7].

In our study, both Rockall and Blatchford scores were calculated in all of the patients on the basis of clinical and endoscopic index variables. Although 30-day mortality rates tended to be higher in patients with a high Rockall score $(\geq 5)$, this tendency was not observed with the Blatchford score in univariate analysis. However, the Blatchford score was originally designed to assess the need for clinical intervention to control bleeding rather than to predict mortality [7]. Notwithstanding, in the multivariate analysis, the Rockall score also failed to show a significant association with 30-day mortality. To clarify predictive factors of NVUGIB-related death, it would be necessary to compare the predictive factors of 30-day mortality according to endoscopic risk stratification. However, our results showed that no factors were independently associated with 30-day mortality according to Forrest classification, probably due to small sample size. Therefore additional large-scale studies are needed to identify predictors associated with 30-day mortality in patients with NVUGIB.

The strengths of this study include its prospective design and inclusion of many factors potentially predictive of mortality in NVUGIB. In addition, the effects of various debated factors on clinical outcomes of patients with NVUGIB were analyzed by examining the data from high-risk patients after adjusting for severity of bleeding. We also attempted to solve the colinearity problem by including Rockall scores $\geq 5$ rather than continuous Rockall scores in the multivariate analysis.

This study had several limitations. First, it was a single-center study with a relatively small sample size, which could lead to sampling bias. Second, the causes of death may have been, to some extent, subjective. However, we assumed that the cause of death, as determined on the basis of imaging findings, clinical symptoms and signs, might have been clinically relevant. Third, although Helicobacter pylori eradication reduces rebleeding in patients with peptic ulcers, we did not test for its presence in our patients [28]. Despite these limitations, our findings provide valuable insight on the outcomes of patients with NVUGIB.

In conclusion, the risk of 30-day mortality in patients with UGIB was significantly higher in patients with advanced age, comorbidity of DM or metastatic malignancy, and hemodynamic instability during hospitalization. These findings suggest that aggressive management and careful monitoring according to specific guidelines should be provided for high-risk patients. Additional prospective studies with a larger number of subjects are needed to support these findings.

\section{KEY MESSAGE}

1. This study demonstrates that comorbidity of diabetes mellitus or metastatic malignancy, age $\geq 65$ years and hemodynamic instability during hospitalization are risk factors of 30 -day mortality in patients with nonvariceal upper gastrointestinal bleeding.

2. Early death was mainly caused by hemorrhage, whereas death after hospital discharge was mainly caused by patients' comorbidities.

\section{Conflict of interest}

No potential conflict of interest relevant to this article was reported.

\section{REFERENCES}

1. Marmo R, Koch M, Cipolletta L, et al. Predictive factors 
of mortality from nonvariceal upper gastrointestinal hemorrhage: a multicenter study. Am J Gastroenterol 2008;103:1639-1647.

2. Holster IL, Kuipers EJ. Management of acute nonvariceal upper gastrointestinal bleeding: current policies and future perspectives. World J Gastroenterol 2012;18:12021207.

3. Gonzalez-Gonzalez JA, Vazquez-Elizondo G, Garcia-Compean $\mathrm{D}$, et al. Predictors of in-hospital mortality in patients with non-variceal upper gastrointestinal bleeding. Rev Esp Enferm Dig 2011;103:196-203.

4. Barkun AN, Bardou M, Kuipers EJ, et al. International consensus recommendations on the management of patients with nonvariceal upper gastrointestinal bleeding. Ann Intern Med 2010;152:101-113.

5. Saltzman JR, Tabak YP, Hyett BH, Sun X, Travis AC, Johannes RS. A simple risk score accurately predicts in-hospital mortality, length of stay, and cost in acute upper GI bleeding. Gastrointest Endosc 2011;74:1215-1224.

6. Rockall TA, Logan RF, Devlin HB, Northfield TC. Risk assessment after acute upper gastrointestinal haemorrhage. Gut 1996;38:316-321.

7. Blatchford O, Murray WR, Blatchford M. A risk score to predict need for treatment for upper-gastrointestinal haemorrhage. Lancet 2000;356:1318-1321.

8. Romagnuolo J, Barkun AN, Enns R, Armstrong D, Gregor J. Simple clinical predictors may obviate urgent endoscopy in selected patients with nonvariceal upper gastrointestinal tract bleeding. Arch Intern Med 2007;167:265-270.

9. Wiley TE, McCarthy M, Breidi L, McCarthy M, Layden TJ. Impact of alcohol on the histological and clinical progression of hepatitis C infection. Hepatology 1998;28:805809.

10. Marin-Gabriel JC, Solis-Herruzo JA. Noninvasive assessment of liver fibrosis: serum markers and transient elastography (FibroScan). Rev Esp Enferm Dig 2009;101:787799.

11. The 86th Congress of the Japanese Society of Legal Medicine. Okayama, Japan. April 17-19, 2002. Abstracts. Nihon Hoigaku Zasshi 2002;56:1-203.

12. Jeon SW, Jung MK, Cho CM, et al. Predictors of immediate bleeding during endoscopic submucosal dissection in gastric lesions. Surg Endosc 2009;23:1974-1979.

13. Forrest JA, Finlayson ND, Shearman DJ. Endoscopy in gastrointestinal bleeding. Lancet 1974;2:394-397.

14. Xu HW, Wang JH, Tsai MS, et al. The effects of cefazolin on cirrhotic patients with acute variceal hemorrhage after endoscopic interventions. Surg Endosc 2011;25:2911-2918.

15. Mungan Z. An observational European study on clinical outcomes associated with current management strategies for non-variceal upper gastrointestinal bleeding (ENERGIB-Turkey). Turk J Gastroenterol 2012;23:463-477.

16. Barkun A, Sabbah S, Enns R, et al. The Canadian Registry on Nonvariceal Upper Gastrointestinal Bleeding and Endoscopy (RUGBE): endoscopic hemostasis and proton pump inhibition are associated with improved outcomes in a real-life setting. Am J Gastroenterol 2004;99:12381246.

17. Crooks C, Card T, West J. Reductions in 28-day mortality following hospital admission for upper gastrointestinal hemorrhage. Gastroenterology 2011;141:62-70.

18. Manguso F, Riccio E, Bennato R, et al. In-hospital mortality in non-variceal upper gastrointestinal bleeding Forrest 1 patients. Scand J Gastroenterol 2008;43:1432-1441.

19. Leontiadis GI, Molloy-Bland M, Moayyedi P, Howden CW. Effect of comorbidity on mortality in patients with peptic ulcer bleeding: systematic review and meta-analysis. Am J Gastroenterol 2013;108:331-345.

20. Nahon S, Hagege H, Latrive JP, et al. Epidemiological and prognostic factors involved in upper gastrointestinal bleeding: results of a French prospective multicenter study. Endoscopy 2012;44:998-1008.

21. Sung JJ, Tsoi KK, Ma TK, Yung MY, Lau JY, Chiu PW. Causes of mortality in patients with peptic ulcer bleeding: a prospective cohort study of 10,428 cases. Am J Gastroenterol 2010;105:84-89.

22. Chiu PW, Ng EK, Cheung FK, et al. Predicting mortality in patients with bleeding peptic ulcers after therapeutic endoscopy. Clin Gastroenterol Hepatol 2009;7:311-316.

23. Ishihara M, Kagawa E, Inoue I, et al. Impact of admission hyperglycemia and diabetes mellitus on short- and longterm mortality after acute myocardial infarction in the coronary intervention era. Am J Cardiol 2007;99:16741679.

24. Cox AJ, Hugenschmidt CE, Wang PT, et al. Usefulness of biventricular volume as a predictor of mortality in patients with diabetes mellitus (from the Diabetes Heart Study). Am J Cardiol 2013;111:1152-1158.

25. Thomsen RW, Riis A, Christensen S, Norgaard M, Sorensen HT. Diabetes and 30-day mortality from peptic ulcer bleeding and perforation: a Danish population-based cohort study. Diabetes Care 2006;29:805-810. 
26. Murata A, Matsuda S, Kuwabara K, Ichimiya Y, Fujino Y, Kubo T. The influence of diabetes mellitus on short-term outcomes of patients with bleeding peptic ulcers. Yonsei Med J 2012;53:701-707.

27. Larche J, Azoulay E, Fieux F, et al. Improved survival of critically ill cancer patients with septic shock. Intensive
Care Med 2003;29:1688-1695.

28. Gisbert JP, Khorrami S, Carballo F, Calvet X, Gene E, Dominguez-Munoz E. Meta-analysis: Helicobacter pylori eradication therapy vs. antisecretory non-eradication therapy for the prevention of recurrent bleeding from peptic ulcer. Aliment Pharmacol Ther 2004;19:617-629. 
Supplementary Table 1. Predictive factors for 30-day mortality in patients with low risk endoscopic stigmata (n = 66)

\begin{tabular}{|c|c|c|c|c|c|}
\hline \multirow[b]{2}{*}{ Factor } & \multicolumn{3}{|c|}{ Univariate analysis } & \multicolumn{2}{|c|}{ Multivariate analysis } \\
\hline & $\begin{array}{l}\text { 30-Day death }(+) \\
(\mathrm{n}=5)\end{array}$ & $\begin{array}{l}\text { 30-Day death }(-) \\
(\mathrm{n}=61)\end{array}$ & $p$ value & Odds ratio (95\% CI) & $p$ value \\
\hline Male sex & $3(60.0)$ & $45(73.8)$ & 0.608 & - & - \\
\hline Age $\geq 65$ yr & $5(100.0)$ & $28(45 \cdot 9)$ & 0.053 & - & - \\
\hline \multicolumn{6}{|l|}{ Initial vital signs } \\
\hline $\mathrm{SBP}<90 \mathrm{mmHg}$ & o & $2(3 \cdot 3)$ & 1.000 & - & - \\
\hline Heart rate $>100$ beat $/ \mathrm{min}$ & o & $15(24.6)$ & 0.581 & - & - \\
\hline \multicolumn{6}{|l|}{ Initial laboratory data } \\
\hline Hemoglobin, g/dL & $6.40 \pm 2.89$ & $8.04 \pm 2.56$ & 0.177 & - & - \\
\hline Platelets, $\times 10^{9} / \mathrm{L}$ & $248 \pm 178$ & $246 \pm 113$ & 0.965 & - & - \\
\hline Blood nitrogen urea, mg/dL & $84.60 \pm 31.92$ & $40.64 \pm 29.19$ & $0.002^{\mathrm{a}}$ & $1.03(0.99-1.08)$ & 0.174 \\
\hline Prothrombin time, sec & $16.02 \pm 10.32$ & $12.43 \pm 3.86$ & 0.481 & - & - \\
\hline $\begin{array}{l}\text { Severe bleeding related symptoms } \\
\text { (hematemesis, hematochezia) }\end{array}$ & $2(40.0)$ & $25(41.0)$ & 1.000 & - & - \\
\hline Positive nasogastric tube aspiration ${ }^{\mathrm{b}}$ & $3(60.0)$ & $29(47.5)$ & 1.000 & - & - \\
\hline Positive rectal examination $^{\mathrm{c}}$ & $5(100.0)$ & $47(77.0)$ & 0.576 & - & - \\
\hline Heavy alcoholics & $2(40.0)$ & $23(37.7)$ & 1.000 & - & - \\
\hline Current smoker & $1(20.0)$ & $23(37.7)$ & 0.645 & - & - \\
\hline Past history of gastrointestinal bleeding & 0 & $17(27.9)$ & 0.317 & - & - \\
\hline Past history of peptic ulcer disease & $1(20.0)$ & $19(31.1)$ & 1.000 & - & - \\
\hline Transfusion requirement & $5(100.0)$ & $40(65.6)$ & 0.169 & - & - \\
\hline Red cell transfusion unit & $2.60 \pm 0.89$ & $1.72 \pm 1.69$ & 0.258 & - & - \\
\hline \multicolumn{6}{|l|}{ Comorbidities } \\
\hline Hypertension & $4(80.0)$ & $30(49.2)$ & 0.357 & - & - \\
\hline Diabetes mellitus & $5(100.0)$ & $17(27.9)$ & $0.003^{\mathrm{a}}$ & $24.79(0.12-81.98)$ & 0.107 \\
\hline Cardiovascular disease & $1(20.0)$ & $13(21.3)$ & 1.000 & - & - \\
\hline Liver cirrhosis & o & $14(23.0)$ & 0.576 & - & - \\
\hline Chronic kidney disease & $2(40.0)$ & $6(9.8)$ & $0.047^{\mathrm{a}}$ & $2.34(0.82-66.62)$ & 0.619 \\
\hline Cerebrovascular disease & $2(40.0)$ & $8(13.1)$ & 0.162 & - & - \\
\hline Heart failure & $3(60.0)$ & $11(18.0)$ & $0.027^{\mathrm{a}}$ & $2.81(0.29-27.21)$ & 0.373 \\
\hline Metastatic malignancy & 0 & $3(4.9)$ & 1.000 & - & - \\
\hline Peripheral vascular disease & $1(20.0)$ & $1(1.6)$ & 0.147 & - & - \\
\hline \multicolumn{6}{|l|}{ Medication } \\
\hline Antiplatelet agents & $2(40.0)$ & $22(36.1)$ & 1.000 & - & - \\
\hline Vitamin $\mathrm{K}$ antagonist & $1(20.0)$ & 0 & 0.076 & - & - \\
\hline Nonsteroidal anti-inflammatory drug & $2(40.0)$ & $13(21.3)$ & 0.318 & - & - \\
\hline PPI comedication & 0 & $2(3 \cdot 3)$ & 1.000 & - & - \\
\hline Blatchford score $\geq 12$ & $5(100.0)$ & $43(70.5)$ & 0.312 & - & - \\
\hline Rockall score $\geq 5$ & $4(80.0)$ & $25(41.0)$ & 0.160 & - & - \\
\hline Endoscopy lesion location & - & - & 1.000 & - & - \\
\hline Body & $1(20.0)$ & $10(16.4)$ & - & - & - \\
\hline Other than body & $4(80.0)$ & $51(83.6)$ & - & - & - \\
\hline $\begin{array}{l}\text { Tachycardia (heart rate }>100 \text { beat } / \mathrm{min} \text { ) } \\
\text { during the admission }\end{array}$ & $2(40.0)$ & $17(27.9)$ & 0.621 & - & - \\
\hline $\begin{array}{l}\text { Hypotension }(\mathrm{SBP}<90 \mathrm{mmHg}) \text { during } \\
\text { the admission }\end{array}$ & $2(40.0)$ & $6(9.8)$ & 0.107 & - & - \\
\hline Rebleeding within 30 days & $1(20.0)$ & $2(3.3)$ & 0.214 & - & - \\
\hline Hospital stay, day & $9.80 \pm 8.44$ & $4.82 \pm 4.95$ & 0.259 & - & - \\
\hline
\end{tabular}

Values are presented as number (\%) or mean \pm SD.

CI, confidence interval; SBP, systolic blood pressure; PPI, proton pump inhibitor.

${ }^{a}$ Significant values with $p<0.05$.

${ }^{\mathrm{b}}$ Nasogastric tube was performed in 64 patients.

${ }^{\mathrm{c}}$ Rectal examination was performed in 66 patients. 
Supplementary Table 2. Predictive factors for 30-day mortality in patients with high-risk endoscopic stigmata $(\mathbf{n}=78)$

\begin{tabular}{|c|c|c|c|c|c|}
\hline \multirow[b]{2}{*}{ Factor } & \multicolumn{3}{|c|}{ Univariate analysis } & \multicolumn{2}{|c|}{ Multivariate analysis } \\
\hline & $\begin{array}{l}\text { 30-Day death }(+) \\
(\mathrm{n}=8)\end{array}$ & $\begin{array}{l}\text { 30-Day death }(-) \\
\quad(\mathrm{n}=70)\end{array}$ & $p$ value & Odds ratio (95\% CI) & $p$ value \\
\hline Male sex & $7(87.5)$ & $58(82.9)$ & 1.000 & - & - \\
\hline Age $\geq 65 \mathrm{yr}$ & $6(75.0)$ & $25(35.7)$ & $0.015^{\mathrm{a}}$ & $39.15(0.72-890.47)$ & 0.121 \\
\hline \multicolumn{6}{|l|}{ Initial vital signs } \\
\hline $\mathrm{SBP}<90 \mathrm{mmHg}$ & o & $8(11.4)$ & 0.591 & - & - \\
\hline Heart rate $>100$ beat $/ \mathrm{min}$ & $3(37.5)$ & $18(25 \cdot 7)$ & 0.675 & - & - \\
\hline \multicolumn{6}{|l|}{ Initial laboratory data } \\
\hline Hemoglobin, g/dL & $8.04 \pm 1.47$ & $8.82 \pm 2.89$ & 0.456 & - & - \\
\hline Platelets, $\times 10^{9} / \mathrm{L}$ & $381 \pm 177$ & $267 \pm 110$ & 0.115 & - & - \\
\hline Blood nitrogen urea, mg/dL & $48.38 \pm 26.84$ & $46.77 \pm 27.75$ & 0.877 & - & - \\
\hline Prothrombin time, sec & $16.98 \pm 13.01$ & $13.30 \pm 9.12$ & 0.306 & - & - \\
\hline $\begin{array}{l}\text { Severe bleeding related symptoms } \\
\text { (hematemesis, hematochezia) }\end{array}$ & $4(50.0)$ & $40(57.1)$ & 0.723 & - & - \\
\hline Positive nasogastric tube aspiration ${ }^{\mathrm{b}}$ & $7(87.5)$ & $56(80.0)$ & 1.000 & - & - \\
\hline Positive rectal examination $^{\mathrm{c}}$ & $8(100.0)$ & $51(72.9)$ & 0.187 & - & - \\
\hline Heavy alcoholics & $3(37 \cdot 5)$ & $26(37.1)$ & 1.000 & - & - \\
\hline Current smoker & $3(37.5)$ & $26(37.1)$ & 1.000 & - & - \\
\hline Past history of gastrointestinal bleeding & $1(12.5)$ & $19(27.1)$ & 0.672 & - & - \\
\hline Past history of peptic ulcer disease & $3(37.5)$ & $30(42.9)$ & 1.000 & - & - \\
\hline Transfusion requirement & $7(87.5)$ & $43(61.4)$ & 0.247 & - & - \\
\hline Red cell transfusion unit & $7.25 \pm 7.03$ & $2.90 \pm 4.53$ & 0.127 & - & - \\
\hline \multicolumn{6}{|l|}{ Comorbidities } \\
\hline Hypertension & $6(75.0)$ & $25(35 \cdot 7)$ & $0.040^{\mathrm{a}}$ & $13.85(0.55-347.77)$ & 0.110 \\
\hline Diabetes mellitus & $3(37.5)$ & $12(17.1)$ & 0.177 & - & - \\
\hline Cardiovascular disease & o & $6(8.6)$ & 1.000 & - & - \\
\hline Liver cirrhosis & o & $8(11.4)$ & 0.591 & - & - \\
\hline Chronic kidney disease & $4(50.0)$ & $11(15.7)$ & $0.040^{\mathrm{a}}$ & $1.66(0.36-75.75)$ & 0.796 \\
\hline Cerebrovascular disease & $3(37.5)$ & $6(8.6)$ & 0.051 & - & - \\
\hline Heart failure & $2(25 \cdot 0)$ & $6(8.6)$ & 0.188 & - & - \\
\hline Metastatic malignancy & $1(12.5)$ & $2(2.9)$ & 0.280 & - & - \\
\hline Peripheral vascular disease & o & $2(2.9)$ & 1.000 & - & - \\
\hline \multicolumn{6}{|l|}{ Medication } \\
\hline Antiplatelet agents & $4(50.0)$ & $18(25 \cdot 7)$ & 0.212 & - & - \\
\hline Vitamin $\mathrm{K}$ antagonist & o & $4(5.7)$ & 1.000 & - & - \\
\hline Nonsteroidal anti-inflammatory drug & $2(25.0)$ & $16(22.9)$ & 1.000 & - & - \\
\hline PPI comedication & 0 & $3(4 \cdot 3)$ & 1.000 & - & - \\
\hline Blatchford score $\geq 12$ & $7(87.5)$ & $45(64 \cdot 3)$ & 0.257 & - & - \\
\hline Rockall score $\geq 5$ & $8(100.0)$ & $50(71.4)$ & 0.105 & - & - \\
\hline Endoscopy lesion location & - & - & 0.275 & - & - \\
\hline Body & $2(25.0)$ & $34(48.6)$ & - & - & - \\
\hline Other than body & $6(75.0)$ & $36(51.4)$ & - & - & - \\
\hline $\begin{array}{l}\text { Tachycardia (heart rate > } 100 \text { beat } / \mathrm{min} \text { ) } \\
\text { during the admission }\end{array}$ & $7(87.5)$ & $22(31.4)$ & $0.003^{\mathrm{a}}$ & $15.80(0.50-500.38)$ & 0.117 \\
\hline $\begin{array}{l}\text { Hypotension }(\mathrm{SBP}<90 \mathrm{mmHg}) \\
\text { during the admission }\end{array}$ & $7(87.5)$ & $21(30.0)$ & $0.003^{\mathrm{a}}$ & $39.01(0.46-3,310.03)$ & 0.106 \\
\hline Rebleeding within 30 days & $6(75.0)$ & $16(22.9)$ & $0.005^{\mathrm{a}}$ & $13.32(0.25-714.42)$ & 0.202 \\
\hline Hospital stay, day & $4.50 \pm 5.43$ & $7.80 \pm 6.19$ & 0.153 & - & - \\
\hline
\end{tabular}

Values are presented as number (\%) or mean $\pm \mathrm{SD}$.

CI, confidence interval; SBP, systolic blood pressure; PPI, proton pump inhibitor.

${ }^{a}$ Significant values with $p<0.05$.

${ }^{\mathrm{b}}$ Nasogastric tube was performed in 76 patients.

${ }^{\mathrm{c}}$ Rectal examination was performed in 76 patients. 